\title{
Norois
}

Environnement, aménagement, société

$247 \mid 2018$

Recherche touristique : perspectives latinoaméricaines

\section{Vers une économie politique du tourisme. Réflexion à partir de quelques expériences latino- américaines}

Towards a political economy of tourism. Reflections on Latin American case studies

Hacia una economía política del turismo. Algunas reflexiones desde América latina

\section{Clément Marie dit Chirot}

\section{OpenEdition}

Journals

Édition électronique

URL : http://journals.openedition.org/norois/6421

DOI : $10.4000 /$ norois.6421

ISBN : 978-2-7535-7629-2

ISSN : 1760-8546

Éditeur

Presses universitaires de Rennes

Édition imprimée

Date de publication : 19 septembre 2018

Pagination : 7-13

ISBN : 978-2-7535-7570-7

ISSN : 0029-182X

Référence électronique

Clément Marie dit Chirot, « Vers une économie politique du tourisme. Réflexion à partir de quelques expériences latino-américaines », Norois [En ligne], 247 | 2018, mis en ligne le 19 septembre 2020, consulté le 04 janvier 2021. URL : http://journals.openedition.org/norois/6421 ; DOI : https://doi.org/ 10.4000 /norois. 6421 


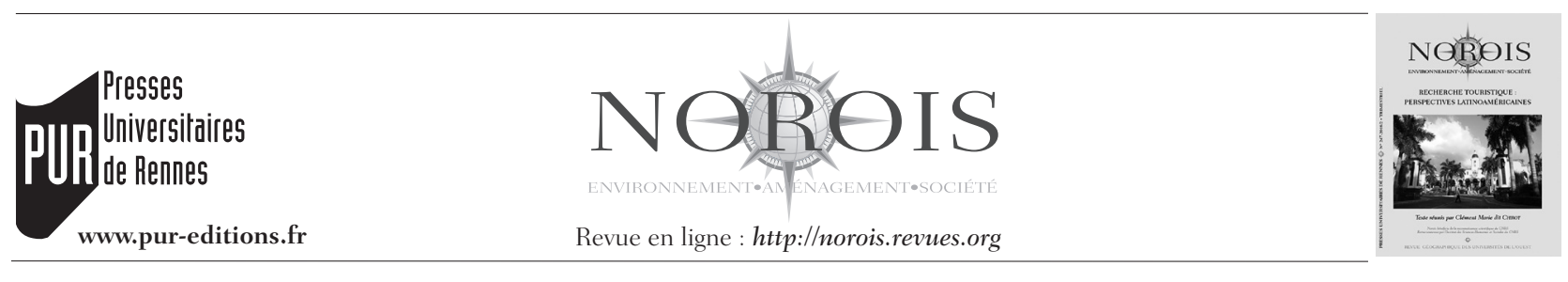

\title{
Introduction \\ Vers une économie politique du tourisme. Réflexion à partir de quelques expériences latino-américaines
}

\author{
Towards a Political Economy of Tourism. Reflections on Latin American Case Studies \\ Hacia una economía política del turismo. Algunas reflexiones desde América latina
}

\author{
Clément Marie dit Chirot
}

Espaces et Sociétés, université d’Angers, Maison des Sciences Humaines - 5bis boulevard Lavoisier, 49045 ANGERS,

France. (clement.marieditchirot@univ-angers.fr)

En l'espace d'une décennie, la recherche latinoaméricaniste sur le tourisme a acquis une visibilité croissante au sein des sciences sociales. Cette évolution s'observe notamment dans le monde académique francophone où plusieurs auteurs soulignaient encore, à la fin des années 2000, la rareté et la dispersion des travaux sur l'Amérique latine dans le champ des études touristiques (Dehoorne et Murat, 2009; Demanget et Dumoulin Kervran, 2010). Au cours des dernières années, cette situation a été en partie compensée par une série de publications scientifiques consacrées aux problématiques touristiques dans le sous-continent. Dans le sillage d'un premier dossier coordonné par la géographe Nathalie Raymond et publié par la revue TRACE en 2004, plusieurs revues régionales ont ainsi fait le choix de consacrer des numéros thématiques à l'étude du fait touristique, à l'instar de la revue Études caribéennes (2009), des Cahiers des Amériques latines (2010), ou encore de la Revue interdisciplinaire de travaux sur les Amériques (2010) dans le contexte éditorial français. Si la question touristique semble avoir gagné en légitimité dans le champ des études latino-américaines, cette évolution est également traduite par un regain d'intérêt pour l'Amérique latine dans le domaine des études touristiques illustré, toujours dans le monde francophone, par le numéro de la revue Téoros consacré à l'Amérique latine (2014) ou par celui de la revue Via Tourism traitant, plus spécifiquement, du cas brésilien $(2015)^{1}$.

Ce dynamisme éditorial est à l'image de celui qu'a connu le phénomène touristique lui-même au cours de la dernière décennie dans la région. Même si le poids de l'Amérique latine dans les échanges touristiques internationaux demeure limité, avec environ $7 \%$ des arrivées internationales enregistrées à l'échelle mondiale en 2016, celle-ci connaît une fréquentation en hausse passée de 64 millions de visi-

1. Ce regain d'intérêt est également matérialisé par plusieurs dossiers thématiques dans des revues anglophones comme Latin American Perspectives (2008) ou la revue Bulletin of Latin American Research (201 1), et par de nombreuses publications dans le monde académique hispanophone. On retiendra notamment l'importance des contributions sur l'Amérique latine au sein de la revue espagnole Pasos et, en Amérique latine, les numéros thématiques de revues comme Desacatos au Mexique (2015), Cuadernos de Geografía en Colombie (2017) ou de la revue péruvienne Apuntes (2018). 
teurs internationaux en 2010 à plus de 90 millions en 2016 selon l'Organisation Mondiale du Tourisme (OMT, 2017). Cette augmentation s'explique en partie par l'accroissement du nombre de touristes états-uniens, favorisé par le cours élevé du dollar et la dépréciation d'un certain nombre de monnaies latino-américaines durant cette période. Un tel accroissement reflète également l'essor des déplacements touristiques intrarégionaux, en lien avec le développement de nouveaux foyers émetteurs et l'accès d'un nombre croissant de Latino-Américains à la pratique du tourisme international. Ces statistiques ne prennent en revanche pas en compte le tourisme national qui, s'il s'avère plus difficilement quantifiable, constitue l'essentiel de l'activité touristique en Amérique latine (Raymond, 2002 ; 2004 ; 2015).

Ces tendances globales ne doivent pas occulter les disparités profondes à l'échelle du sous-continent. Le Mexique concentrait en effet à lui seul près de $40 \%$ des arrivées internationales en 2016, une proportion atteignant $54 \%$ si l'on ajoute la République Dominicaine, Cuba et Porto Rico. À l'inverse, la place du Brésil dans les échanges touristiques internationaux reste faible au regard du poids économique et démographique du pays, mais aussi de l'importance du marché touristique intérieur (Théry, 2015). La situation est également contrastée en Amérique centrale où le Costa Rica s'affirme comme un haut-lieu de l'écotourisme à l'échelle internationale (Raymond, 2007; Boukhris, 2016) tandis que le Honduras ou le Salvador demeurent à l'écart des principaux circuits touristiques. Malgré cette hétérogénéité, le développement du tourisme concerne un nombre croissant d'espaces urbains ou ruraux et suscite des enjeux particulièrement vifs sur le plan économique, politique ou environnemental. Que l'on pense aux logiques d'intégration économique régionale à l'œuvre dans l'isthme centraméricain ou aux recompositions de la société cubaine depuis la "Période Spéciale ${ }^{2}$ ", à la patrimonialisation des quartiers centraux de nombreuses villes ou encore à l'urbanisation rapide de certains littoraux, l'étude du tourisme donne à lire les dynamiques contemporaines des sociétés latino-américaines dont

2. Le terme désigne à Cuba la période succédant à la chute du Mur de Berlin et à l'effondrement du système soviétique. La disparition de ce soutien au niveau international marque l'entrée en crise du système économique instauré avec la Révolution cubaine et le début d'une série de changements structurels marquée, notamment, par le développement rapide du tourisme international. elle révèle les permanences et les mutations, les contradictions anciennes ou récentes, les tensions parfois violentes. Si le tourisme constitue en cela un «prisme d'observation » des sociétés du souscontinent (Raymond, 2004), l'Amérique latine offre en retour un terrain d'étude particulièrement riche pour interroger le fait touristique dans ses multiples dimensions à travers des questionnements transposables à d'autres régions du Monde, au moins dans les sociétés du Sud. Les contributions ici réunies ont donc en commun d'éclairer certaines réalités latino-américaines contemporaines, mais aussi de s’inscrire dans les débats théoriques plus généraux que connaît aujourd'hui la recherche touristique.

\section{LA DÉPENDANCE EN HÉRITAGE? L'AMÉRIQUE LATINE AU PRISME DE QUELQUES DÉBATS CONTEMPORAINS SUR LE TOURISME DANS LES SUDS}

Interroger le fait touristique à partir de ses manifestations latino-américaines revêt un intérêt particulier à l'aune des débats qui ont animé les études touristiques depuis l'émergence de ce champ de recherche au début des années 1970. Cette spécificité tient notamment à la manière dont les réalités sociales du sous-continent ont alimenté l'approche scientifique du tourisme lorsque le phénomène s'inscrit dans les pays du Sud. Tandis que la diffusion du tourisme s'accélère dans un certain nombre de pays en développement à partir des années 1960, la problématique de "l'impact» du tourisme sur les sociétés réceptrices s’impose comme paradigme dominant pour penser l'essor du phénomène en dehors des sociétés occidentales où l'activité touristique a vu le jour. Le cadre théorique privilégié multiplie alors les emprunts conceptuels à la théorie de la dépendance, principal courant du marxisme latino-américain dont l'influence marque de nombreux travaux sur les sociétés en développement à cette époque. Face aux discours des organismes internationaux considérant le tourisme comme le nouveau " moteur de développement du TiersMonde » (Duterme, 2006), de nombreux chercheurs insistent sur les asymétries économiques dont participe le développement du phénomène à l'échelle mondiale. Car si le tourisme international peut certes contribuer à la redistribution de la richesse entre pays industrialisés et pays en développement, 
l'activité n'échappe pas aux rapports inégaux entre les différentes régions du Monde mais s’inscrit au contraire dans une trajectoire historique propre à de nombreuses sociétés du Tiers-Monde, en lien avec la dynamique du « système-monde » (Wallerstein, 1979). Bien qu'un tel cadre conceptuel ne soit pas toujours revendiqué explicitement, la parenté théorique entre la problématique de l'impact et la théorie de la dépendance transparaît dans les termes utilisés, telle l'opposition entre centre et périphérie, le recours aux concepts de "sous-développement ", de «Tiers-Monde », ou l'assimilation fréquente du tourisme à une nouvelle forme de colonialisme.

L'approche du tourisme fondée sur la théorie de l'impact comportait de nombreux écueils, parmi lesquels celui de présenter des sociétés du Sud passives face à un phénomène exogène contrôlé depuis les pays riches (Michaud, 2001 ; Doquet, 2010). La prise en compte des inégalités entre Nord et Sud s'est ainsi souvent effectuée au détriment de la complexité des sociétés réceptrices et de leurs logiques internes, négligeant l'étude des jeux d'acteurs à l'échelle locale. La sensibilité aux inégalités NordSud s'est par ailleurs souvent focalisée sur le clivage supposé entre touristes et populations locales, censé incarner les relations asymétriques entre « les sociétés nanties des pays développés » (Cazes, 1992) et celles de pays pauvres convertis en «périphéries du plaisir » (Turner et Ash, 1975). Cette opposition se cristallise notamment autour du couple conceptuel visiteurs/visités (host/guest), longtemps considéré comme la principale contradiction traversant les sociétés touristiques dans les pays du Sud. En limitant l'approche du tourisme à sa dimension internationale, cette approche a également conduit à exclure de l'analyse les pratiques touristiques des populations des Suds elles-mêmes, dont les travaux plus récents ont montré l'importance, mais également l'ancienneté.

L'approche critique du tourisme contenue dans la théorie de l'impact est aujourd'hui tombée en désuétude. Il faut dire qu'en adossant l'étude du tourisme à sa dénonciation presque systématique et en raison de la vision réductrice du phénomène qu'elle véhiculait, l'entrée par la question de l'impact prêtait le flan à un certain nombre de critiques légitimes. À ce titre, on retiendra que Georges Cazes pointait déjà au début des années 1990 les limites d'un tel cadre d'analyse et la nécessité d'un renouvellement épistémologique (Cazes, 1992). Ce renouvellement a effectivement eu lieu. Pour certains chercheurs, il s'est traduit par un décentrement du regard et un intérêt croissant pour les pratiques touristiques des populations issues des sociétés du Sud (Sacareau et al., 2015). Dans d'autres cas il passe par une prise de distance avec les analyses structuralistes et marxistes, privilégiant une lecture micro-sociologique jugée plus à même de restituer la complexité et l'ambivalence des relations de pouvoir en contexte touristique (Roux, 2011; Simoni, 2008). Cette réflexion alimente plus largement les débats actuels sur le dépassement des lectures classiques des rapports Nord-Sud, inspirés par certaines approches post-coloniales.

Les débats que connaissent les études touristiques ont donc montré la nécessité de faire évoluer nos cadres d'analyse. Mais cette nécessité de renouvellement a semble-t-il parfois fourni un prétexte pour liquider, un peu hâtivement, l'héritage de pensées critiques importantes. À juste titre, Magali Demanget et David Dumoulin-Kervran pointent d'ailleurs le risque de " passer de l'archétype du tout passivité menant à la destruction culturelle, au tout résistance et tout stratégie, négligeant alors les contraintes structurelles inégalitaires » (2010). Il semble en effet que la critique des approches structuralistes et marxistes du tourisme ait parfois été excessive, conduisant, en quelque sorte, à « jeter le bébé » (à savoir une sensibilité particulière aux formes de domination économique dont participe le développement du tourisme) avec « l'eau du bain » (la problématique de l'impact). Pourtant, les limites de la problématique de l'impact reflètent davantage la manière dont la théorie de la dépendance a été appliquée à l'étude du tourisme à partir d'une lecture simplifiée des travaux dépendantistes. Car si ce courant a pu donner lieu à des interprétations caricaturales réduisant la dynamique des sociétés du Sud aux effets d'une domination extérieure exercée depuis les pays riches, l'apport fondamental de la théorie de la dépendance a été d'éclairer les rapports sociaux internes aux sociétés dépendantes, notamment en Amérique latine. La prise en compte de l'histoire coloniale et l'analyse des formes spécifiques d'insertion dans la division internationale du travail qui en résulte ont en effet permis d'appréhender des configurations sociales propres aux formes périphériques de capitalisme, traduites 
par des niveaux d'inégalités sociales exacerbés ou encore par le poids des questions ethnico-raciales dans la définition des hiérarchies sociales. En d'autres termes, "le caractère principal de l'interprétation par la dépendance n'est pas l'étude des relations internationales (qui ne doit cependant pas être oubliée), mais l'analyse des classes sociales au sein du capitalisme dépendant » (Bresser-Pereira, 2009). Si l'on ne saurait évidemment pas prôner un retour aux lectures manichéennes développées dans les années 1970, la recherche sur le tourisme dans les Suds gagnerait donc peut-être aujourd'hui à revisiter un cadre théorique dont le philosophe Enrique Dussel rappelle par ailleurs l'actualité et la pertinence pour penser les inégalités liées à la globalisation (Dussel, 2009; 2014).

\section{DES DYNAMIQUES TOURISTIQUES HÉTÉROGÈNES À L'ÉCHELLE RÉGIONALE}

Au croisement d'approches et d'horizons disciplinaires différents, les contributions réunies dans ce dossier permettent précisément d'interroger à nouveaux frais ces débats sur les évolutions contemporaines des sociétés latino-américaines. L'article du géographe Ernest Cañada montre comment l'Amérique centrale a émergé comme « nouvelle périphérie touristique » à partir des années 1990. Si l'essor du tourisme a permis de diversifier une économie régionale jusqu'alors très dépendante des activités agro-exportatrices, celui-ci s’est opéré conformément à l'orientation néolibérale préconisée par les grands bailleurs de fonds internationaux. Dans ce contexte, les formes de mise en tourisme observées s'accompagnent souvent d'une subordination accrue des travailleurs locaux sur le marché de l'emploi et de logiques prédatrices vis-à-vis des ressources naturelles, deux tendances matérialisées par la multiplication des conflits socio-environnementaux à l'échelle locale. Des processus similaires sont analysés par Samuel Jouault dont le texte met en perspective deux études de cas situées dans la péninsule du Yucatan, au Mexique, et dans la baie de Tela au Honduras. Dans ces deux espaces marqués par la présence historique de populations indigènes ou afro-descendantes, mayas au Mexique ou garifunas au Honduras, l'auteur met en évidence des mécanismes d'éviction spatiale au profit d'agents extérieurs. Il nuance cependant les visions dualistes opposant des communautés locales sans défense face à l'intrusion d'acteurs exogènes. L'article montre en effet que cette dépossession peut être favorisée par des conflits intra-communautaires préexistants ou s'effectuer avec le concours de leaders locaux ou d'une partie des élites locales. Le développement du tourisme peut alors contribuer à accentuer les inégalités sociales localement, comme semble en témoigner l'apparition de nouvelles formes de ségrégation socio-spatiale.

La question des peuples autochtones, incontournable en Amérique latine, est également au centre de l'article de Mónica Martínez Mauri sur le Panama. Dans la région de Gunayala, le développement du tourisme est depuis plusieurs années l'enjeu d'un conflit opposant les populations Kunas au gouvernement panaméen pour le contrôle de la manne touristique. Alors que les Kunas bénéficient historiquement d'une autonomie importante vis-àvis de l'État, s'exprimant notamment à travers le pouvoir qu'ont les autorités traditionnelles Kunas de prélever certaines formes d'impôts, ce droit ancestral est aujourd'hui remis en cause lorsqu'il s'applique aux acteurs du tourisme. À travers l'étude des conflits pour le contrôle de la fiscalité touristique, l'anthropologue analyse les relations complexes entre le peuple Kuna et l'État panaméen. L'article montre ainsi la persistance de rapports de domination hérités de la période coloniale et les formes de colonialité du pouvoir (Quijano, 2000) traversant, aujourd'hui encore, l'État et la société panaméenne.

L'analyse des rapports sociaux au sein des sociétés touristiques d'Amérique latine doit donc prendre en compte l'articulation complexe entre des logiques sociales opérant à différentes échelles géographiques. Cette nécessité apparaît de manière claire dans l'article de Gustavo Marín Guardado sur le Mexique. À Tulum, sur l'un des littoraux touristiques les plus convoités de la Caraïbe mexicaine, le phénomène de spoliation foncière particulièrement violent qui affecte l'ejido Pino Suarez n'est pas le fait de multinationales étrangères mais de puissants investisseurs du nord du Mexique agissant avec la complicité des autorités locales. Par l'analyse fine des jeux d'acteurs à l'échelle locale, l'auteur met en évidence l'existence de «mafias agraires » et le rôle central de ce phénomène dans les logiques plus 
globales de l' « accumulation par dépossession » (Harvey, 2010). Cette complexité apparaît enfin dans l'article de Tristan Loloum et Antonio Aledo sur lequel s'ouvre ce dossier. En retraçant le boom immobilier-touristique qu'a connu le Nordeste brésilien entre le début des années 2000 et la crise financière de 2008, les auteurs montrent les liens entre ce processus et la dynamique internationale du capitalisme à cette période marquée par l'émergence de bulles immobilières. L'article montre cependant que les rapports de forces économiques sur le marché de l'immobilier sont souvent moins univoques que ne le suggèrent certaines analyses globalisantes issues, notamment, de la géographie radicale anglophone. Dans un contexte institutionnel peu lisible pour les investisseurs internationaux, ces derniers sont en effet tributaires d'acteurs locaux - publics ou privés - dont le rôle central dans le développement touristique invite à relativiser une interprétation en termes de "dépendance » ou de domination Nord-Sud.

À différents niveaux, les processus touristiques analysés dans ce numéro sont révélateurs des évolutions profondes qu'a connues l'Amérique latine depuis les années 1960 et 1970, époque à laquelle ont vu le jour la théorie de la dépendance et la problématique de l'impact. Et si la perspective régionale qui sous-tend le dossier laisse entrevoir un certain nombre de régularités et de problématiques communes, ces évolutions sont également traduites par une diversification des situations à l'échelle nationale et locale. Comme le remarque Robert Boyer, on aurait effectivement tort de penser que « l'appartenance à une même zone géographique impliquerait une communauté des modes de développement » ou de minorer la présence de «trajectoires nationales extrêmement contrastées » (Boyer, 2012). Malgré la persistance difficilement contestable d'asymétries profondes à l'échelle mondiale, il importe donc de distinguer des « degrés de périphéricité » (Bernard et al., 2017) et de se garder de toute interprétation homogénéisante. Si les différents points de vue développés par les auteurs du dossier témoignent de cette diversité, ils expriment par ailleurs un pluralisme théorique dont l'enjeu dépasse le contexte régional latino-américain. Ce pluralisme interroge plus largement la manière dont les sciences sociales, et en particulier les approches critiques, se saisissent aujourd'hui de la question touristique.

\section{VERS UN RENOUVELLEMENT DE L'APPROCHE CRITIQUE DU TOURISME?}

Si l'étude du tourisme constitue une entrée particulièrement riche pour appréhender les réalités sociales du sous-continent, les expériences latino-américaines appellent en retour une série de questionnements plus généraux sur les cadres théoriques mobilisés par la recherche touristique au-delà du contexte régional. En se confrontant à des contextes sociaux figurant parmi les plus inégalitaires au monde, la recherche touristique peut en effet difficilement faire l'économie d'une réflexion sur les rapports de classes au sein des sociétés touristiques. Ce questionnement prend une acuité particulière dans un domaine de recherche fortement influencé par le tournant culturel et par les théories post-structuralistes depuis le début des années 1990 (Bianchi, 2009; Hiernaux, 2008; Marie dit Chirot, 2017). Ces évolutions théoriques sont d'autant plus visibles qu'elles s'accompagnent, comme l'ont montré les travaux du sociologue Bertrand Réau, du poids croissant des sciences de gestion au sein des études touristiques. Comme le souligne ce dernier, ce mouvement se traduit par le déclin des approches critiques dans le champ des études touristiques et par la définition d'« un espace du pensable en adéquation avec les intérêts économiques, politiques et sociaux des acteurs du tourisme » (Réau, 2017). Dans ce contexte, « les rares travaux qui mettent en question le tourisme lui-même en interrogeant les rapports de pouvoir qui s'y jouent représentent les fractions les plus périphériques du domaine de la recherche sur le tourisme » (ibid.).

Ce contexte académique éclaire le choix de réunir dans ce numéro des contributions plaçant au centre de l'analyse l'étude des contradictions sociales au sein des sociétés touristiques. Les textes ici présentés ont en commun de proposer une lecture matérialiste du fait touristique et de ramener la réflexion sur le tourisme dans le champ de l'économie politique ${ }^{3}$. Ce dossier s'inscrit en cela dans une proximité conceptuelle avec

3. Sans entrer dans les nombreux débats sur la définition de ce courant, nous faisons ici référence à une approche accordant une attention particulière à la dimension économique des faits sociaux mais admettant, comme le remarque la sociologue Béatrice Hibou, que « les contours de l'économie ne sont pas définis à l'avance et que «l'invention de l'économique » résulte du processus complexe lié aussi bien à la construction de l'État national qu'à la réalité sociale et à l'exercice disciplinaire du pouvoir ». Comme elle le rappelle, «l'économie politique est aussi une science empirique, une science du réel ou plutôt une science de la « réalité historique », de

«l'homme et des conduites de vie » concrètes, de «l'homme vivant » et de 
des recherches menées depuis plusieurs années dans les contextes anglophone (Bianchi, 2009; 2017) et hispanophone (Cañada et Murray, à paraître) mais encore relativement rares en France. Ce choix n'implique cependant pas une uniformité sur le plan théorique comme en témoigne, par exemple, les rapports différenciés qu'entretiennent les auteurs avec la géographie radicale. Alors que le texte d'Ernest Cañada mobilise les travaux de David Harvey pour montrer le rôle de la diffusion spatiale du tourisme dans la résolution des crises structurelles du capitalisme, l'article de Tristan Loloum et Antonio Aledo pointe les limites d'une approche surplombante postulant l'existence de déterminations causales trop directes entre la dynamique globale du capitalisme et les processus touristiques observés localement. Pour appréhender les relations entre investisseurs internationaux et acteurs locaux, les auteurs privilégient une analyse en termes d'« homologies structurales » (Bourdieu, 1992; Dezalay et Garth, 2002) et insistent sur les relations tissées entre des acteurs occupant des positions comparables au sein de structures sociales étroitement liées aux contextes nationaux. Ces différences d'interprétations montrent l'importance d'un dialogue entre théories critiques et entre les différentes traditions disciplinaires issues des sciences sociales. Le croisement entre la géographie et l'anthropologie autour duquel s'articule le dossier - à l'exception de la note de recherche de l'historienne Elda Moreno Acevedo sur le développement de l'aviation touristique dans la péninsule du Yucatan - est d'ailleurs facilité par le rapport privilégié qu'entretiennent les auteurs avec la démarche ethnographique. Cette dimension empirique est indissociable du positionnement théorique qui anime le numéro et rappelle que « le système-monde n'est pas un cadre holiste, théoriquement constitué, qui fournirait un contexte aux enquêtes ethnographiques, mais un contexte qui émerge, s'intègre et se distribue, pièce par pièce, dans les objets discontinus de l'ethnographie multisituée » (Marcus, 2010).

\section{LE CONFLIT COMME OBJET, L'ESPACE TOURISTIQUE COMME ENJEU}

À la lumière de ces enjeux théoriques, les processus analysés dans ce dossier font apparaître

la « vie subjective individuelle » qui, par définition, comprend de multiples dimensions » (Hibou, 2011). différentes pistes susceptibles de contribuer au renouvellement de l'approche critique du tourisme. Ce renouvellement pourrait avoir comme point de départ un questionnement sur les conflits inhérents aux logiques de mise en tourisme, dont la multiplication est particulièrement visible en Amérique latine. À ce titre, l'article d'Ernest Cañada invite à une réflexion systématique sur les « conflits touristiques » et rappelle que les épisodes de crise ou de conflit sont souvent des moments privilégiés pour l'étude des dynamiques sociales. Qu'il s'agisse de conflits redistributifs portant sur les conditions de travail, de tensions foncières, de controverses environnementales ou encore de revendications d'autonomie exprimées par des populations autochtones, l'entrée par le conflit permet d'entrevoir des lignes de tensions au sein des sociétés touristiques et met en lumière des enjeux et des mécanismes habituellement peu visibles. Abordé sous cet angle, le tourisme cesse d'apparaître comme un phénomène dont il faudrait étudier les effets - positifs ou négatifs - sur les espaces et les sociétés, mais plutôt comme une arène dans laquelle s'exercent des relations de pouvoir et de domination opérant à différentes échelles d'espace et de temps.

Enfin, les processus analysés dans le dossier sont marqués par le poids des enjeux spatiaux dans les dynamiques touristiques. Cette dimension concerne évidemment l'espace physique lorsque celui-ci est l'objet de luttes foncières ou quand le développement touristique s'accompagne d'une fermeture croissante de l'espace résidentiel. Les processus présentés montrent par ailleurs que les luttes autour de la production et de l'appropriation de l'espace touristique ne se résument pas à sa dimension matérielle et englobent différents niveaux de spatialité. La dimension spatiale du fait touristique interroge en effet la capacité qu'ont les acteurs et les groupes sociaux de contrôler la manne touristique par le biais de la fiscalité ou encore de maitriser les logiques de valorisation symbolique à l'origine de bulles immobilières. Ces différentes problématiques s'articulent au sein d'une réflexion plus générale sur la dimension spatiale du tourisme : Qui contrôle l'espace touristique? Qui en est exclu? Dans quelle mesure les luttes pour l'espace touristique contribuent-elles à la reproduction, ou au contraire, à la redéfinition des hiérarchies sociales? L'attention portée par les auteurs à cette question témoigne, au-delà de la géographie, de l'intérêt croissant des sciences sociales pour la dimen- 
sion spatiale des sociétés. Elle pointe sans doute également la manière particulière dont l'activité touristique mobilise l'espace en contribuant à la valorisation économique et symbolique de lieux jadis peu convoités, plaçant ces derniers au centre d'enjeux économiques, politiques et sociaux particulièrement vifs en Amérique latine comme dans d'autres régions du Monde.

\section{Remerciements}

Ce dossier thématique a été mené à bien avec le soutien de la région Pays de la Loire dans le cadre du programme Angers TourismLab.

\section{Bibliographie}

Bernard N., Blondy C., Duhamel P., 2017, Tourisme et périphéries. La centralité des lieux en question, Rennes, PUR.

Bianchi R., 2017, The political economy of mass tourism and its contradictions, in Harrison D., Sharpley R. (dir.), Mass tourism in a Small World, Wallingford, CAB International, p. $40-52$.

Bianchi R., 2009, The "Critical Turn" in Tourism Studies: a Radical Critique, Tourism Geographies, vol. 11, no 4, p. 484-504.

Boukhris L., 2016, La fabrique circulatoire d'un patrimoine national ou la coproduction de la nature au Costa-Rica, Autrepart, no 78-79, p. 257-275.

Boundieu P., 1992, Les règles de l'art : genèse et structure du champ littéraire, Paris, Le Seuil.

Boyer R., 2012, Diversité et évolution des capitalismes en Amérique latine. De la régulation économique au politique, Revue de la Régulation, $\mathrm{n}^{\circ} 11$.

Bresser-Pereira L.C., 2009, Amérique latine : de l'interprétation nationaliste à l'interprétation par la dépendance, Revue Tiers-Monde, no 199, p. 533-546.

Cañada E., Murray I. (à paraître), Perspectivas críticas en turismo, Tenerife, Pasos Edita.

Dehoorne O., Murat C., 2009, Regards croisés sur les enjeux du tourisme en Amérique latine, Études Caribéennes, n 13-14.

Dezalay Y., Garth B., 2002, La mondialisation des guerres de palais. La restructuration du pouvoir d'État en Amérique latine. Entre notables du droit et «Chicago Boys », Paris, Le Seuil.

Demanget M., Dumoulin-Kervran D., 2010, Étudier le tourisme : vers de nouveaux horizons, Cahiers des Amériques latines, $n^{\circ} 65$, p. 19-33.

Doquet A., 2010, La force de l'impact. Paradigme théorique et réalités de terrain, Espacestemps.net.

Duterme B., 2006, Expansion du tourisme international: gagnants et perdants, vol. XIII, Paris, CETRI-Syllepse, coll. «Alternatives Sud».
Dussel E., 2009, De la philosophie de la libération. Entretien avec Enrique Dussel, Cahiers des Amériques latines, nº 62, p. $37-46$.

Dussel E., 2014, 16 Tesis de economía política, Mexico, Siglo XXI.

Harvey D., 2010, Le nouvel impérialisme, Paris, Les Prairies Ordinaires.

Hibou B., 2011 , Anatomie politique de la domination, Paris, La Découverte.

Hiernaux D., 2008, El giro cultural y las nuevas interpretaciones geográficas del turismo, Espaço e Tempo, nº 23, p. 177-187.

Marcus G.E., 2010, Ethnographie du/dans le système-monde. L'émergence d'une ethnographie multisituée, in CEFAÏ D. (dir.), L'engagement ethnographique, Paris, Éditions de l'École des hautes études en sciences sociales, p. 371-395.

Marie dit Chirot C., 2017, Rematérialiser les études touristiques, in Guibert C., Taunay B. (dir.), Tourisme et sciences sociales. Postures de recherches, ancrages disciplinaires et épistémologiques, Paris, L'Harmattan, coll. «Logiques sociales ».

Michaud J., 2001, Anthropologie, tourisme et sociétés locales au fil des textes, Anthropologie et sociétés, vol. 25, n 2, p. 15-33.

Омт, 2017, Faits saillants du tourisme, Organisation Mondiale du Tourisme.

Quijano A., 2000, Colonialidad del poder, eurocentrismo y América latina, in LANDER E. (dir.), La colonialidad del saber: eurocentrismo y ciencias sociales, Buenos Aires, CLACSO.

Raymond N., 2002, Tourisme national et international dans les pays andins : quelles relations? L'exemple du Pérou, Bulletin de l'Institut français d'études andines, $\mathrm{n}^{\circ} 31$ (1), p. 23-38.

Raymond N., 2004, Los interrogantes que plantea América Latina al estudio del fenómeno turístico, TRACE, n ${ }^{\circ} 45$, p. 11-31.

Raymond N., 2007, Costa Rica : du petit pays « démocratique, sain et pacifique », au leader de l'écotourisme et de la protection de l'environnement, Études Caribéennes, nº 6.

Raymond N., 2015, Les pratiques de tourisme et de loisirs des Latino-Américains chez eux : quelles spécificités?, in Sacareau I., Taunay B., Peyvel E. (dir.), La mondialisation du tourisme. Les nouvelles frontières d'une pratique, Rennes, PUR, p. 29-42.

Reau B., 2017, Esquisses pour une analyse critique des tourism studies, Zilsel, n², p. 223-249.

Roux S., 2011, No money, no honey. Économies intimes du tourisme sexuel en Thaïlande, Paris, La Découverte.

Sacareau I., Taunay B., Peyvel E., 2015, La mondialisation du tourisme. Les nouvelles frontières d'une pratique, Rennes, PUR.

Simoni V., 2008, Shifting Power. The (de)stabilization of asymmetries in the realm of tourism in Cuba, Tsansta: Review of the Swiss Anthropological Society, no 13, p. 11-19.

THÉRY H., 2015, Lieux et flux du tourisme intérieur brésilien, Via Tourism Review, $\mathrm{n}^{\circ} 7$.

Turner L., Ash J., 1975, The Golden Hordes : International Tourism and the Pleasure Periphery, Londres, Constable \& Co.

Wallerstein I., i979, The Capitalist World-Economy, Cambridge, Cambridge University Press. 